\title{
A quadratic inequality for sum of co-adjoint orbits
}

\author{
Naichung Conan Leung and Xiaowei Wang
}

\begin{abstract}
We obtain an effective lower bound on the distance of the sum of co-adjoint orbits from the origin. Even when the distance is zero (thus the symplectic quotient is well defined) our result gives a nontrivial constraint on these co-adjoint orbits. In the particular case of unitary groups, we obtain the quadratic inequality for eigenvalues of Hermitian matrices satisfying
\end{abstract}

$$
A+B=C
$$

This quadratic inequality can be interpreted as the Chern number inequality for semi-stable reflexive toric sheaves.

\section{Introduction}

Given any rank $r$ Hermitian matrix $A$, we may order its eigenvalues in such a way that

$$
\lambda_{1}(A) \geq \lambda_{2}(A) \geq \cdots \geq \lambda_{r}(A)
$$

and denote $\lambda(A):=\left(\lambda_{1}(A), \ldots, \lambda_{r}(A)\right) \in \mathbb{R}^{r}$ as its spectrum. In [6] (also see [3] for an excellent account of the subject), Klyachko discovered following series of linear inequalities for Hermitian matrices $A, B, C$ satisfying $A+B=C$ :

$$
\sum_{k \in K} \lambda_{k}(C) \leq \sum_{i \in I} \lambda_{i}(A)+\sum_{j \in J} \lambda_{j}(B)
$$

for all triple of subsets $I, J, K \subset\{1,2, \ldots, r\}$ of the same cardinality and such that the associated Schubert cycle $s_{K}$ is a component of $s_{I} \cdot s_{J}$. This result can be interpreted as describing the linear inequalities that determine the intersection of the sum of co-adjoint orbits $\mathcal{O}_{\lambda(A)}+\mathcal{O}_{\lambda(B)}+\mathcal{O}_{\lambda(-C)}$ with the positive Weyl chamber for the unitary group, which is a convex polytope by Kirwan's convexity theorem. Klyachko's result was generalized to any compact Lie group by Berenstein and Sjamaar in the beautiful paper [2], they are able to relate above eigenvalue inequalities to the convexity of the image of the moment map. All above inequalities are linear on eigenvalues of matrices. 
In [7], we find a natural quadratic inequality on these eigenvalues by relating the Hermitian matrices to stable reflexive sheaves over the projective spaces (c.f. [5]). More precisely, we have found the following

Theorem 1.1. Suppose $A_{1}, \ldots, A_{N}$ are rank $r$ Hermitian matrices satisfying

$$
\sum_{\alpha=1}^{N} A_{\alpha}=0 .
$$

Then the following inequality holds true;

$$
\sum_{\alpha=1}^{N} \lambda\left(A_{\alpha}\right)^{2} \leq \sum_{1 \leq \alpha \neq \beta \leq N} \lambda\left(A_{\alpha}\right) \lambda\left(-A_{\beta}\right) .
$$

Moreover, the equality holds if and only if all but two of $A_{\alpha}$ 's are scalar matrices.

We have explained in [7] the relationship between this quadratic inequality and the classical Chern number inequality for Gieseker polystable vector bundles over a projective manifold (not necessarily being toric). Our dream is to find a universal "Chern number inequality" for stable objects in the sense of geometric invariant theory such that the classical Chern number inequality for vector bundles will be a consequence. In order to do so, we made our first step, that is, we extend the above results to the case of sum of co-adjoint orbits for ANY compact Lie group.

Main Theorem. Let $\mathcal{O}_{\lambda_{\alpha}}^{\prime}$ s be co-adjoint orbits of a compact Lie group $G$ with $\alpha=1,2, \ldots, N$. If we choose $\lambda_{\alpha}$ be the unique point of the intersection of $\mathcal{O}_{\lambda_{\alpha}}$ with the positive Weyl chamber. Then for any $\lambda$ in the sum of co-adjoint orbits

$$
\lambda \in \sum_{\alpha=1}^{N} \mathcal{O}_{\lambda_{\alpha}}
$$

we have

$$
|\lambda|^{2} \geq \sum_{\alpha=1}^{N}\left|\lambda_{\alpha}\right|^{2}+\sum_{\alpha \neq \beta}\left\langle\lambda_{\alpha}, w_{0} \cdot \lambda_{\beta}\right\rangle
$$

where $w_{0}$ is the longest element in the Weyl group $W$.

Furthermore, the equality holds if and only if all except at most two of $\lambda_{\alpha}^{\prime} s, 1 \leq \alpha \leq N$ are in the center of $\mathfrak{g}$. 
Notice that $\sum_{\alpha=1}^{N} \mathcal{O}_{\lambda_{\alpha}} \subset \mathfrak{g}^{*}$ is the image of the moment map for the diagonal $G$-action on $\prod_{\alpha=1}^{N} \mathcal{O}_{\lambda_{\alpha}}$. If we want to construct the symplectic quotient $\left(\prod_{\alpha=1}^{N} \mathcal{O}_{\lambda_{\alpha}}\right) / / G$, we need the origin to be inside of the image of moment map. Thus our main theorem gives a necessary constraint for this to happen, namely $\sum_{\alpha=1}^{N}\left|\lambda_{\alpha}\right|^{2}+\sum_{\alpha \neq \beta}\left\langle\lambda_{\alpha}, w_{0} \cdot \lambda_{\beta}\right\rangle$ must be nonpositive.

Suppose $\sum_{\alpha=1}^{N}\left|\lambda_{\alpha}\right|^{2}+\sum_{\alpha \neq \beta}\left\langle\lambda_{\alpha}, w_{0} \cdot \lambda_{\beta}\right\rangle$ is positive, then our main theorem gives an effective lower bound on the distance between $\sum_{\alpha=1}^{N} \mathcal{O}_{\lambda_{\alpha}}$ and the origin in $\mathfrak{g}^{*}$. If we define

$$
\begin{aligned}
r^{2} & =\sum_{\alpha=1}^{N}\left|\lambda_{\alpha}\right|^{2}+\sum_{\alpha \neq \beta}\left\langle\lambda_{\alpha}, w_{0} \cdot \lambda_{\beta}\right\rangle \\
R^{2} & =\sum_{\alpha=1}^{N}\left|\lambda_{\alpha}\right|^{2}+\sum_{\alpha \neq \beta}\left\langle\lambda_{\alpha}, \lambda_{\beta}\right\rangle=\left|\sum_{\alpha=1}^{N} \lambda_{\alpha}\right|^{2} .
\end{aligned}
$$

Then we have

$$
\sum_{\alpha=1}^{N} \mathcal{O}_{\lambda_{\alpha}} \subset B(R)-B(r) \subset \mathfrak{g}^{*}
$$

where $B(R)$ and $B(r)$ are balls centered at origin of radii $R$ and $r$, respectively. As it was found in [7], that Equation (1.1) is closely related to the Chern number inequality for stable vector bundles, the main motivation of the generalization made in this paper is to obtain the analogy of Chern number inequality for the zeros of moment map.

Finally, let us close this section by introducing our notations for the remaining sections.

$G ; T ; Z$

$\mathfrak{g} ; \mathfrak{t} ; \mathfrak{z}$

$\langle\cdot, \cdot\rangle$

$\mathfrak{t}_{+}$

$\mathfrak{W} ; w_{0}$

$\left(\mathcal{O}_{\lambda}, \Omega_{\mathcal{O}_{\lambda}}\right) \subset \mathfrak{g}$

$\mathfrak{c}_{\lambda} \subset \mathfrak{g}$

$\mathfrak{c}_{\lambda}^{\mathrm{SS}}, \mathfrak{z}_{\lambda} \subset \mathfrak{c}_{\lambda}$ compact Lie group; its maximal torus; its center

Lie algebra of $G ; T ; Z$

bi-invariant inner product on $\mathfrak{g}$, which identifies $\mathfrak{g}$

with $\mathfrak{g}^{*}$

positive Weyl chamber

Weyl group of $G$; a longest element in the Weyl group

adjoint orbit through $\lambda \in \mathfrak{t}_{+}$with $\Omega_{\mathcal{O}_{\lambda}}$ being its

symplectic form

centralizer of $\lambda \in \mathfrak{t}$

semi-simple part of $\mathfrak{c}_{\lambda}$, center of $\mathfrak{c}_{\lambda}$ 
$C_{\lambda}, C_{\lambda}^{\text {ss }} \subset G \quad$ closed subgroups of $G$ with Lie algebra $\mathfrak{c}_{\lambda}$ and $\mathfrak{c}_{\lambda}^{\text {ss }}$

$(\cdot)^{\top}: \mathfrak{g} \rightarrow \mathfrak{z}_{\lambda} \quad$ orthogonal projection to $\mathfrak{z}_{\lambda}$ with respect to $\langle\cdot, \cdot\rangle_{\mathfrak{g}}$

$(\cdot)^{\perp}: \mathfrak{g} \rightarrow \mathfrak{z}_{\lambda}^{\perp} \quad$ projection to the orthogonal completement of $\mathfrak{z} \lambda$ with respect to $\langle\cdot, \cdot\rangle_{\mathfrak{g}}$

\section{Convexity of moment map}

Let us consider the diagonal $G$-action on the symplectic manifold $\prod_{\alpha=1}^{N} \mathcal{O}_{\lambda_{\alpha}}$ with Kostant-Kirillov-Souriau symplectic form, then the map

$$
\begin{gathered}
i^{*}: \prod_{\alpha=1}^{N} \mathfrak{g} \longrightarrow \mathfrak{g} \\
\left(\xi_{1}, \ldots, \xi_{N}\right) \longrightarrow \sum_{\alpha=1}^{N} \xi_{\alpha}
\end{gathered}
$$

being dual to the diagonal embedding $i: G \hookrightarrow G^{N}$ is the moment map of the diagonal $G$-action. We define

$$
\Delta_{N}:=i^{*}\left(\prod_{\alpha=1}^{N} \mathcal{O}_{\lambda_{\alpha}}\right) \cap \mathfrak{t}_{+}
$$

i.e., $\Delta_{N}$ is the moment polytope inside the positive Weyl chamber. If we restrict the $G$-action to its maximal torus, then the moment map for the $T$-action is given by $\Pi:=\left.j^{*} \circ i^{*}\right|_{\prod_{\alpha=1}^{N} \mathcal{O}_{\lambda_{\alpha}}}$, where $j^{*}: \mathfrak{g} \rightarrow \mathfrak{t}$ is the projection induced from $j: T \rightarrow G$. That is,

$$
\begin{aligned}
& \Pi: \prod_{\alpha=1}^{N} \mathcal{O}_{\lambda_{\alpha}} \longrightarrow \mathfrak{t} \\
& \left(\xi_{1}, \ldots, \xi_{N}\right) \longrightarrow j^{*}\left(\sum_{\alpha=1}^{N} \xi_{\alpha}\right) .
\end{aligned}
$$

In this section, we prove

Theorem 2.1. For any $\lambda \in \Delta_{N}=i^{*}\left(\prod_{\alpha=1}^{N} \mathcal{O}_{\lambda_{\alpha}}\right) \cap \mathfrak{t}_{+}$, we have

$$
\left\langle\lambda, \sum_{\alpha=1}^{N} \lambda_{\alpha}\right\rangle \geq|\lambda|^{2}
$$


Moreover, the equality holds if and only if

$$
\lambda=\sum_{\alpha=1}^{N} \lambda_{\alpha} \quad \text { or } \quad \lambda=0
$$

The proof of the above theorem based on the convexity property of moment maps. Let us first state the following elementary lemma and the proof will be left to the readers.

Lemma 2.1. For any $\lambda, \eta \in \mathfrak{t}_{+}$, we have

$$
\left\langle\lambda, w_{0} \cdot \eta\right\rangle \leq\langle\lambda, w \cdot \eta\rangle \leq\langle\lambda, \eta\rangle
$$

for any $w \in \mathfrak{W}$. Moreover, we have

1. $\langle\lambda, \eta\rangle=\left\langle\lambda, w_{0} \cdot \eta\right\rangle$ if and only if $\eta$ or $\lambda \in \mathfrak{z}$.

2. If $\lambda$ lies in the interior of $\mathfrak{t}_{+}$then $\left\langle\lambda, w_{0} \cdot \eta\right\rangle=\langle\lambda, w \cdot \eta\rangle$ if and only if $w_{0} \cdot \eta=w \cdot \eta$.

The next lemma describes the convex hull $\operatorname{Hull}(\mathfrak{W} \cdot \lambda)$ of a Weyl group orbit.

Lemma 2.2. Suppose $\lambda, \eta \in \mathfrak{t}_{+}$satisfying $\langle\lambda, \zeta\rangle=\langle\eta, \zeta\rangle$ for any $\zeta \in \mathfrak{z}$ and $\langle\xi, \lambda\rangle \geq\langle\xi, \eta\rangle$ for any $\xi \in \mathfrak{t}_{+}$, then

$$
\eta \in \operatorname{Hull}(\mathfrak{W} \cdot \lambda)
$$

Proof. First, we have a Lie algebra decomposition $\mathfrak{g}=\mathfrak{z} \oplus \mathfrak{z}^{\perp}$. By our assumption $\langle\lambda, \zeta\rangle=\langle\eta, \zeta\rangle$ for any $\zeta \in \mathfrak{z}$ and the fact that $\mathfrak{z}$ is invariant under $\mathfrak{W}$, we may reduce to the case that $\mathfrak{z}=0$. By assumption $\langle\xi, \lambda-\eta\rangle \geq 0$ for any $\xi \in \mathfrak{t}_{+}$, precisely means that $\eta$ always lies in one side of the supporting hyperplane of the convex set Hull $(\mathfrak{W} \cdot \lambda) \subset \mathfrak{t}$, hence

$$
\eta \in \operatorname{Hull}(\mathfrak{W} \cdot \lambda)
$$

Let us recall the following convexity theorem due to Atiyah [1], Guillemin and Sternberg [4]. 
Theorem 2.2. Let $(X, \omega)$ be a symplectic manifold with a Hamiltonian $T$-action such that fixed points are all isolated and $\mu: X \longrightarrow \mathfrak{t}$ be its moment map. Then

$$
\operatorname{Im}(\mu)=\operatorname{Hull}\left(\left\{c_{1}, \ldots, c_{p}\right\}\right)
$$

with $\left\{c_{i}\right\}$ 's being the fixed points of the T-action.

In order to apply the above theorem, we restrict our $G$-action to its maximal torus, then the moment map is given by $\Pi:=\left.j^{*} \circ i^{*}\right|_{\prod_{\alpha=1}^{N} \mathcal{O}_{\lambda_{\alpha}}}$, where $j^{*}: \mathfrak{g} \rightarrow \mathfrak{t}$ is the map induced from $j: T \rightarrow G$. That is,

$$
\begin{aligned}
& \Pi: \prod_{\alpha=1}^{N} \mathcal{O}_{\lambda_{\alpha}} \longrightarrow \\
& \left(\xi_{1}, \ldots, \xi_{N}\right) \quad \longrightarrow \quad j^{*}\left(\sum_{\alpha=1}^{N} \xi_{\alpha}\right) .
\end{aligned}
$$

Then we obtain

Theorem 2.3.

$$
\operatorname{Im} \Pi=\operatorname{Hull}\left(\mathfrak{W} \cdot \sum_{\alpha=1}^{N} \lambda_{\alpha}\right)
$$

where $\mathfrak{W} \cdot\left(\sum_{\alpha=1}^{N} \lambda_{\alpha}\right)$ is the orbit of $\sum_{\alpha=1}^{N} \lambda_{\alpha}$ under the action of $\mathfrak{W}$. Furthermore, we have

$$
\Pi^{-1}\left(w \cdot \sum_{\alpha=1}^{N} \lambda_{\alpha}\right) \in \prod_{\alpha=1}^{N} \mathfrak{t}
$$

for any $w \in \mathfrak{W}$.

Proof. First, we notice that the fixed points of the adjoint action of $T$ on $\mathfrak{g}$ is $\mathfrak{t}$, this implies that the image of the fixed point set under $\Pi$ is

$$
\left\{\sum_{\alpha=1}^{N} w_{\alpha} \cdot \lambda_{\alpha} \in \mathfrak{t} \mid\left(w_{1}, \ldots, w_{N}\right) \in \mathfrak{W}^{N}\right\} .
$$

By Lemma 2.1, we have

$$
\left\langle\sum_{\alpha=1}^{N} \lambda_{\alpha}, \xi\right\rangle=\sum_{\alpha=1}^{N} \max _{w \in \mathfrak{W}}\left\langle w \cdot \lambda_{\alpha}, \xi\right\rangle=\max _{\left(w_{1}, \ldots, w_{N}\right) \in \mathfrak{W}^{N}}\left\langle\sum_{\alpha=1}^{N} w_{\alpha} \cdot \lambda_{\alpha}, \xi\right\rangle
$$


for $\xi \in \mathfrak{t}_{+}$. This implies that, for any $\xi \in \mathfrak{t}_{+}$

$$
\left\langle\sum_{\alpha=1}^{N} \lambda_{\alpha}, \xi\right\rangle \geq\left\langle\sum_{\alpha=1}^{N} w_{\alpha} \cdot \lambda_{\alpha}, \xi\right\rangle
$$

So we deduce

$$
\operatorname{Hull}\left(\mathfrak{W} \cdot \sum_{\alpha=1}^{N} \lambda_{\alpha}\right) \ni \sum_{\alpha=1}^{N} w_{\alpha} \cdot \lambda_{\alpha}
$$

for any $\left(w_{1}, \ldots, w_{N}\right) \in \mathfrak{W}^{N}$ by Lemma 2.2. Combining this with Theorem 2.2 , we have

$$
\operatorname{Im} \Pi=\operatorname{Hull}\left(\sum_{\alpha=1}^{N} w_{\alpha} \cdot \lambda_{\alpha} \mid\left(w_{1}, \ldots, w_{N}\right) \in \mathfrak{W}^{N}\right)=\operatorname{Hull}\left(\mathfrak{W} \cdot \sum_{\alpha=1}^{N} \lambda_{\alpha}\right)
$$

Corollary 2.1. Suppose $\lambda \in \Delta_{N}$. Then for any $0 \neq \eta \in \mathfrak{t}_{+}$, we have

$$
\left\langle\eta, \sum_{\alpha=1}^{N} \lambda_{\alpha}\right\rangle \geq\langle\eta, \lambda\rangle
$$

Moreover, the equality holds if and only if $\lambda=\sum_{\alpha=1}^{N} \lambda_{\alpha}$.

Proof of Theorem 2.1. Let $\eta=\lambda$ then the inequality follows from the above corollary.

\section{Proof of the main result}

By using the co-adjoint action of $G$, it is enough for us to prove the inequality for $\lambda \in \Delta_{N}$. Since we are going to prove the main theorem by induction, for any positive integer $N$, we introduce the following statement $\left(*_{N}\right)$ to indicate its dependence on $N$.

Statement $\left(*_{N}\right)$. Let $G$ be a compact Lie group and $\left\{\lambda_{1}, \ldots, \lambda_{N}\right\} \subset \mathfrak{t}_{+}$. For any $\lambda \in \Delta_{N}$, we have

$$
|\lambda|^{2} \geq \sum_{\alpha=1}^{N}\left|\lambda_{\alpha}\right|^{2}+\sum_{\alpha \neq \beta}\left\langle\lambda_{\alpha}, w_{0} \cdot \lambda_{\beta}\right\rangle
$$


with the equality holds if and only if there are $w_{1}, \ldots, w_{N} \in \mathfrak{W}$, the Weyl group of $G$, such that

$$
\lambda:=\sum_{\alpha=1}^{N} w_{\alpha} \cdot \lambda_{\alpha}
$$

and

$$
\left\langle\lambda_{\alpha}, w_{0} \cdot \lambda_{\beta}\right\rangle=\left\langle w_{\alpha} \cdot \lambda_{\alpha}, w_{\beta} \cdot \lambda_{\beta}\right\rangle \text { for } 1 \leq \alpha, \beta \leq N .
$$

Before we proceed let us verify that the existence of $w_{1}, \ldots, w_{N} \in \mathfrak{W}$ with

$$
\left\langle\lambda_{\alpha}, w_{0} \cdot \lambda_{\beta}\right\rangle=\left\langle w_{\alpha} \cdot \lambda_{\alpha}, w_{\beta} \cdot \lambda_{\beta}\right\rangle \quad \text { for } 1 \leq \alpha, \beta \leq N
$$

is equivalent to the condition that all but at most two of $\lambda_{\alpha}$ 's are in $\mathfrak{z}$.

For simplicity, let us assume that $w_{N}=\mathrm{id}$ and $\lambda_{N}$ is semisimple, that is, it is not fixed by any element in $\mathfrak{W}$. The identities

$$
\left\langle\lambda_{N}, w_{0} \cdot \lambda_{\alpha}\right\rangle=\left\langle\lambda_{N}, w_{\alpha} \cdot \lambda_{\alpha}\right\rangle \quad \text { for } 1 \leq \alpha \leq N-1
$$

and the semisimplicity of $\lambda_{N}$ imply $w_{0} \cdot \lambda_{\alpha}=w_{\alpha} \cdot \lambda_{\alpha}$ for $1 \leq \alpha \leq N-1$ due to Lemma 2.1. Hence for $1 \leq \alpha, \beta \leq N-1$, we have

$$
\left\langle\lambda_{\alpha}, w_{0} \cdot \lambda_{\beta}\right\rangle=\left\langle w_{\alpha} \cdot \lambda_{\alpha}, w_{\beta} \cdot \lambda_{\beta}\right\rangle=\left\langle w_{0} \cdot \lambda_{\alpha}, w_{0} \cdot \lambda_{\beta}\right\rangle=\left\langle\lambda_{\alpha}, \lambda_{\beta}\right\rangle .
$$

By Lemma 2.1 again, this implies that all but at most one of $\lambda_{\alpha}$ 's, $1 \leq \alpha \leq$ $N-1$ lie in $\mathfrak{z}$. For general $\lambda_{N}$, the idea is very similar, and we leave it to the readers.

Now our main theorem stated in the introduction is equivalent to the following

Theorem 3.1. Statement $\left(*_{N}\right)$ holds true for any positive integer $N$.

To make the proof more transparent, let us briefly sketch the main idea modulo the technical details. As we mentioned earlier, we will argue by mathematical induction on $N$. In Proposition 3.1 we show that if $\lambda=0$ then the inequality

$$
0 \geq \sum_{\alpha=1}^{N}\left|\lambda_{\alpha}\right|^{2}+\sum_{\alpha \neq \beta}\left\langle\lambda_{\alpha}, w_{0} \cdot \lambda_{\beta}\right\rangle
$$

follows from statement $\left(*_{N-1}\right)$. So the argument boils down to reducing the general case to the case of $\lambda=0$. To do that, we first show in Lemma 3.1 that 
when $|\lambda|$ attain its minimum, its necessary that $\lambda \in \mathfrak{g}_{\xi}$ where $\xi \in i^{*-1}(\lambda)$. Then by introducing a smaller group $C_{\lambda}^{\mathrm{ss}} \subset G$ which is essentially perpendicular to $\lambda$, we will be able to reduce the problem to the case with $\lambda=0$ but a smaller group $C_{\lambda}^{\text {ss }} \subset G$ to which the Proposition 3.1 is applicable, hence we have finished the proof.

Proposition 3.1. Suppose statement $\left(*_{N-1}\right)$ holds true, then we have

$$
0 \geq \sum_{\alpha=1}^{N}\left|\lambda_{\alpha}\right|^{2}+\sum_{\alpha \neq \beta}\left\langle\lambda_{\alpha}, w_{0} \cdot \lambda_{\beta}\right\rangle
$$

provided $0 \in \Delta_{N}$. Moreover, the equality holds if and only if $\lambda_{\alpha}=0$ for $1 \leq$ $\alpha \leq N$ or there is a $\lambda_{\nu} \neq 0$ such that

$$
\left(1-w_{0}\right) \cdot \lambda_{\nu}=\sum_{\alpha=1}^{N} \lambda_{\alpha}
$$

and

$$
\left\langle\lambda_{\alpha}, w_{0} \cdot \lambda_{\beta}\right\rangle=\left\langle\lambda_{\alpha}, \lambda_{\beta}\right\rangle \text { for } \alpha, \beta \neq \nu
$$

that is, all but two of $\lambda_{\alpha}$ 's are in $\mathfrak{z}$.

Proof. Without loss of generality, we may assume $\lambda_{N} \neq 0$. Our assumption $\lambda=0 \in \Delta_{N}$ implies $-w_{0} \cdot \lambda_{N} \in \Delta_{N-1}$. Then statement $\left(*_{N-1}\right)$ says

$$
\left|-w_{0} \cdot \lambda_{N}\right|^{2} \geq \sum_{\alpha=1}^{N-1}\left|\lambda_{\alpha}\right|^{2}+\sum_{\substack{\alpha \neq \beta \\ 1 \leq \alpha, \beta \leq N-1}}\left\langle\lambda_{\alpha}, w_{0}\left(\lambda_{\beta}\right)\right\rangle .
$$

On the other hand, Theorem 2.1 implies

$$
\left\langle-w_{0} \cdot \lambda_{N}, \sum_{\alpha=1}^{N-1} \lambda_{\alpha}\right\rangle \geq\left|-w_{0} \cdot \lambda_{N}\right|^{2}
$$

By adding up above inequalities, we obtain

$$
0 \geq \sum_{\alpha=1}^{N}\left|\lambda_{\alpha}\right|^{2}+\sum_{\substack{\alpha \neq \beta \\ 1 \leq \alpha, \beta \leq N}}\left\langle\lambda_{\alpha}, w_{0}\left(\lambda_{\beta}\right)\right\rangle
$$


which is precisely what we want. Moreover, by Theorem 2.1 the equality holds if and only if

$$
-w_{0} \cdot \lambda_{N}=\sum_{\alpha=1}^{N-1} \lambda_{\alpha}
$$

since $\lambda_{N} \neq 0$, and statement $\left(*_{N-1}\right)$ implies that equality holds if and only if there are $\left\{w_{\alpha}\right\}_{\alpha=1}^{N} \subset \mathfrak{W}$ satisfying

$$
-w_{0} \cdot \lambda_{N}:=\sum_{\alpha=1}^{N-1} w_{\alpha} \cdot \lambda_{\alpha}
$$

and

$$
\left\langle\lambda_{\alpha}, w_{0} \cdot \lambda_{\beta}\right\rangle=\left\langle w_{\alpha} \cdot \lambda_{\alpha}, w_{\beta} \cdot \lambda_{\beta}\right\rangle \text { for } 1 \leq \alpha, \beta \leq N-1 .
$$

In particular, we have $\sum_{\alpha=1}^{N-1} w_{\alpha} \cdot \lambda_{\alpha}=\sum_{\alpha=1}^{N-1} \lambda_{\alpha}$, which is only possible if $w_{\alpha} \cdot \lambda_{\alpha}=\lambda_{\alpha}$ for $1 \leq \alpha \leq N-1$ by Lemma 2.1 . So the equality holds if and only if

$$
-w_{0} \cdot \lambda_{N}=\sum_{\alpha=1}^{N-1} \lambda_{\alpha}
$$

and

$$
\left\langle\lambda_{\alpha}, w_{0} \cdot \lambda_{\beta}\right\rangle=\left\langle\lambda_{\alpha}, \lambda_{\beta}\right\rangle \text { for } 1 \leq \alpha, \beta \leq N-1
$$

which is possible only if all but at most one of $\lambda_{\alpha}, 1 \leq a \leq N-1$ are in $\mathfrak{z}$.

Thus all we need to do is to reduce the general case to the $\lambda=0$ case, in order to do so we need some preparation from Lie theory and moment map theory.

Lemma 3.1. Suppose $\min _{\eta \in \Delta_{N}}|\eta|^{2}$ is attained by $\lambda \in \Delta_{N}$. Then for any $\xi \in i^{*-1}(\lambda) \subset \prod_{\alpha=1}^{N} \mathcal{O}_{\lambda_{\alpha}}$ we have $\lambda \in \mathfrak{g}_{\xi}$, the stabilizer of $\xi$ under the $G$-action.

Proof. Notice that the minimum of $|\eta|^{2}$ over $\Delta_{N}=i^{*}\left(\prod_{\alpha} \mathcal{O}_{\lambda_{\alpha}}\right) \cap \mathfrak{t}_{+}$and $i^{*}\left(\prod_{\alpha} \mathcal{O}_{\lambda_{\alpha}}\right)$ are the same because of the Ad-invariance of the metric $|\cdot|$ on $\mathfrak{g}$. Hence $\xi$ is a critical point for the norm squared of the moment map $i^{*}$ for the $G$-action on $\prod_{\alpha} \mathcal{O}_{\lambda_{\alpha}}$. By general theory of moment map [4], $\lambda=i^{*}(\xi)$ must lie inside stabilizer of $\xi$.

From now on, let us assume $|\lambda|^{2}=\min _{\eta \in \Delta_{N}}|\eta|^{2}$ and $\xi:=\left(\xi_{1}, \ldots, \xi_{N}\right) \in$ $\prod_{\alpha=1}^{N} \mathcal{O}_{\lambda_{\alpha}}$ satisfying $\lambda=i^{*}(\xi) \in \Delta_{N}$. By Lemma 3.1 we have $\lambda \in \mathfrak{g}_{\xi}$, which 
is equivalent to

$$
\left[\lambda, \xi_{\alpha}\right]=0 \quad \text { for } 1 \leq \alpha \leq N
$$

That is, $\xi_{\alpha} \in \mathfrak{c}_{\lambda}$, the Lie algebra of the centralizer $C_{\lambda} \subset G$ of $\lambda$.

In order to reduce our considerations to the $\lambda=0$ case, we introduce the Lie algebra decomposition

$$
\mathfrak{c}_{\lambda}=\mathfrak{c}_{\lambda}^{\mathrm{ss}} \oplus \mathfrak{z} \lambda
$$

where $\mathfrak{c}_{\lambda}^{\text {ss }} \subset \mathfrak{c}_{\lambda}$ is the semi-simple part and $\mathfrak{z}_{\lambda} \subset \mathfrak{c}_{\lambda}$ is the center. Correspondingly, for $\forall \xi \in \mathfrak{c}_{\lambda}$, we may write $\xi=\xi^{\perp}+\xi^{\top}$ with $\xi^{\perp} \in \mathfrak{c}_{\lambda}^{\text {ss }}$ and $\xi^{\top} \in \mathfrak{z} \lambda$, note that this is an orthogonal decomposition of $\xi$ with respect to the bi-invariant inner product. Let $C_{\lambda}^{\mathrm{ss}}$ be the closed subgroup of $G$ with Lie algebra $\mathfrak{c}_{\lambda}^{\mathrm{ss}}$ and $\mathcal{O}_{\lambda_{\alpha}^{\perp}}$ 's be the $C_{\lambda}^{\text {ss }}$-orbits of $G$-action restricted to $C_{\lambda}^{\text {ss }}$. Then

$$
\left(\xi_{1}^{\perp}, \ldots, \xi_{N}^{\perp}\right) \in \prod_{\alpha=1}^{N} \mathcal{O}_{\lambda_{\alpha}^{\perp}}=\prod_{\alpha=1}^{N} \mathcal{O}_{\lambda_{\alpha}} \cap\left(\mathfrak{c}_{\lambda}^{\mathrm{sS}}\right)^{N} \subset \mathfrak{g}^{N}
$$

and

$$
\left(\left.i^{*}\right|_{\mathfrak{c}_{\lambda}^{\mathrm{ss} *}}\right)^{\perp}: \prod_{\alpha=1}^{N} \mathcal{O}_{\lambda_{\alpha}^{\perp}} \longrightarrow \mathfrak{c}_{\lambda}^{\mathrm{ss} *}
$$

is exactly the moment map of the $C_{\lambda}^{\mathrm{ss}}$-action on $\prod_{\alpha=1}^{N} \mathcal{O}_{\lambda_{\alpha}^{\perp}}$. This implies

$$
\left(\left.i^{*}\right|_{\mathfrak{c}_{\lambda}^{\mathrm{ss} *}}\right)^{\perp}\left(\xi_{1}^{\perp}, \ldots, \xi_{N}^{\perp}\right)=\sum_{\alpha=1}^{N} \xi_{\alpha}^{\perp}=\lambda^{\perp}=0
$$

and

$$
\lambda=\left(\sum_{\alpha=1}^{N} \xi_{\alpha}\right)^{\top}=\sum_{\alpha=1}^{N} \xi_{\alpha}^{\top}
$$

because $\lambda \in \mathfrak{z} \lambda$. Since the maximal torus of $C_{\lambda}$ is a subtori of $T$, there are $g_{\alpha} \in C_{\lambda}^{\text {ss }}$ for $1 \leq \alpha \leq N$ such that $\eta_{\alpha}:=A d_{g_{\alpha}} \xi_{\alpha} \in \mathfrak{t}_{\lambda,+}$, the positive Weyl chamber for $C_{\lambda}$. However $\mathfrak{t}_{\lambda,+}$ in general is not a subset of $\mathfrak{t}_{+}$, in any case there are $w_{\alpha} \in \mathfrak{W}$ such that $\eta_{\alpha}=w_{\alpha} \cdot \lambda_{\alpha}$. For any $\eta \in \mathfrak{z} \lambda$ of unit length, 
we have

$$
\begin{aligned}
\left\langle\xi_{\alpha}, \eta\right\rangle \eta & =\left\langle\operatorname{Ad}_{g_{\alpha}^{-1}} \eta_{\alpha}, \eta\right\rangle \eta \\
& =\left\langle\eta_{\alpha}, \operatorname{Ad}_{g_{\alpha}} \eta\right\rangle \eta \\
& =\left\langle\eta_{\alpha}, \eta\right\rangle \eta
\end{aligned}
$$

which means

$$
\sum_{\alpha=1}^{N} \eta_{\alpha}^{\top}=\sum_{\alpha=1}^{N} \xi_{\alpha}^{\top}=\lambda
$$

The following lemma helps us to reduce the general case to $\lambda=0$ case.

Lemma 3.2. Let $\lambda$ be as above and $w \in \mathfrak{W}_{C_{\lambda}^{\mathrm{ss}}}$, the Weyl group of $C_{\lambda}^{\mathrm{ss}}$. Then

$$
\sum_{\alpha=1}^{N}\left|\eta_{\alpha}\right|^{2}+\sum_{\alpha \neq \beta}\left\langle\eta_{\alpha}, w \cdot \eta_{\beta}\right\rangle-|\lambda|^{2}=\sum_{\alpha \neq \beta}\left\langle\eta_{\alpha}^{\perp}, w \cdot \eta_{\beta}^{\perp}\right\rangle+\sum_{\alpha=1}^{N}\left|\eta_{\alpha}^{\perp}\right|^{2} .
$$

Proof. The assumption $w \in \mathfrak{W}_{C_{\lambda}^{\text {ss }}}$ implies that $w \cdot \zeta=\zeta$ for any $\zeta \in \mathfrak{z}_{\lambda}$. Hence we have $\langle w \cdot \eta, \zeta\rangle=\langle\eta, \zeta\rangle$ for any $\eta \in \mathfrak{t}, \zeta \in \mathfrak{z}_{\lambda}$, thus $(w \cdot \eta)^{\top}=\eta^{\top}$, or equivalently $((1-w) \cdot \eta)^{\top}=0$. Moreover, $w \cdot \eta^{\top}=(w \cdot \eta)^{\top}$ since $w$ fixes $\mathfrak{z}_{\lambda}$. Therefore, $w \cdot \eta^{\perp}=(w \cdot \eta)^{\perp}$.

Let $\Lambda:=\sum_{\alpha=1}^{N} \eta_{\alpha}$, we calculate, using above formulae and (3.2)

$$
\begin{aligned}
& \sum_{\alpha=1}^{N}\left|\eta_{\alpha}\right|^{2}+\sum_{\alpha \neq \beta}\left\langle\eta_{\alpha}, w \cdot \eta_{\beta}\right\rangle \\
& \quad=\sum_{\alpha=1}^{N}\left\langle\eta_{\alpha},(1-w) \cdot \eta_{\alpha}\right\rangle+\langle\Lambda, w \cdot \Lambda\rangle \\
& \quad=\sum_{\alpha=1}^{N}\left\langle\eta_{\alpha}^{\perp},(1-w) \cdot \eta_{\alpha}^{\perp}\right\rangle+\left\langle\Lambda^{\perp}+\Lambda^{\top}, w \cdot\left(\Lambda^{\perp}+\Lambda^{\top}\right)\right\rangle \\
& \quad=\sum_{\alpha=1}^{N}\left|\eta_{\alpha}^{\perp}\right|^{2}-\sum_{\alpha=1}^{N}\left\langle\eta_{\alpha}^{\perp}, w \cdot \eta_{\alpha}^{\perp}\right\rangle+\left|\Lambda^{\top}\right|^{2}+\left\langle\Lambda^{\perp}, w \cdot \Lambda^{\perp}\right\rangle \\
& \quad=\sum_{\alpha=1}^{N}\left|\eta_{\alpha}^{\perp}\right|^{2}-\sum_{\alpha=1}^{N}\left\langle\eta_{\alpha}^{\perp}, w \cdot \eta_{\alpha}^{\perp}\right\rangle+|\lambda|^{2}+\left\langle\Lambda^{\perp}, w \cdot \Lambda^{\perp}\right\rangle .
\end{aligned}
$$


Proof of Theorem 3.1. We prove the theorem by applying mathematical induction on $N$.

For $N=1$, the statement is trivially true. We assume now statement $\left(*_{N-1}\right)$ holds true, and take $G$ to be $C_{\lambda}^{\text {ss }}$ then Proposition 3.1 says precisely

$$
\sum_{\alpha \neq \beta}\left\langle\eta_{\alpha}^{\perp},-w_{0}^{C_{\lambda}^{\mathrm{ss}}} \cdot \eta_{\beta}^{\perp}\right\rangle \geq \sum_{\alpha=1}^{N}\left|\eta_{\alpha}^{\perp}\right|^{2}
$$

where $w_{0}^{C_{\lambda}^{\mathrm{ss}}}$ is the longest element of $\mathfrak{W}_{C_{\lambda}^{\mathrm{ss}}}$. Moreover, the equality holds if and only if $\eta_{\alpha}^{\perp}=0$ for all $1 \leq \alpha \leq N$ or $\eta_{N}^{\perp} \neq 0$

$$
\begin{aligned}
0 \neq-w_{0}^{C_{\lambda}^{\mathrm{ss}}} \eta_{N}^{\perp} & =\sum_{\alpha=1}^{N-1} \eta_{\alpha}^{\perp} \text { and }\left\langle\eta_{\alpha}^{\perp}, \eta_{\beta}^{\perp}\right\rangle \\
& =\left\langle\eta_{\alpha}^{\perp}, w_{0}^{C_{\lambda}^{\mathrm{ss}}} \cdot \eta_{\beta}^{\perp}\right\rangle \quad \text { for } 1 \leq \alpha, \beta \leq N-1 .
\end{aligned}
$$

By applying Lemma 3.2 we see that inequality (3.4) is equivalent to

$$
\begin{aligned}
|\lambda|^{2} & \geq \sum_{\alpha=1}^{N}\left|\eta_{\alpha}\right|^{2}+\sum_{\alpha \neq \beta}\left\langle\eta_{\alpha}, w_{0}^{C_{\lambda}^{\mathrm{ss}}} \cdot \eta_{\beta}\right\rangle \\
& =\sum_{\alpha=1}^{N}\left|w_{\alpha} \cdot \lambda_{\alpha}\right|^{2}+\sum_{\alpha \neq \beta}\left\langle w_{\alpha} \cdot \lambda_{\alpha}, w_{0}^{C_{\lambda}^{\mathrm{ss}}} \cdot w_{\beta} \cdot \lambda_{\beta}\right\rangle .
\end{aligned}
$$

Since $\mathfrak{W}$ acts on $\mathfrak{t}$ isometrically, we have $\left|w_{\alpha} \cdot \lambda_{\alpha}\right|^{2}=\left|\lambda_{\alpha}\right|^{2}$ and

$$
\left\langle w_{\alpha} \cdot \lambda_{\alpha}, w_{0}^{C_{\lambda}^{\mathrm{ss}}} \cdot w_{\beta} \cdot \lambda_{\beta}\right\rangle=\left\langle\lambda_{\alpha}, w_{\alpha}^{-1} \cdot w_{0}^{C_{\lambda}^{\mathrm{ss}}} \cdot w_{\beta} \cdot \lambda_{\beta}\right\rangle \geq\left\langle\lambda_{\alpha}, w_{0} \cdot \lambda_{\beta}\right\rangle
$$

by Lemma 2.1. These imply that

$$
|\lambda|^{2} \geq \sum_{\alpha=1}^{N}\left|\lambda_{\alpha}\right|^{2}+\sum_{\alpha \neq \beta}\left\langle\lambda_{\alpha}, w_{0} \cdot \lambda_{\beta}\right\rangle .
$$

Moreover, it follows from (3.5) that for the equality to hold in the above inequality, one needs $\left\langle\eta_{\alpha}, w_{0}^{C_{\lambda}^{\mathrm{ss}}} \cdot \eta_{\beta}\right\rangle=\left\langle w_{\alpha} \cdot \lambda_{\alpha}, w_{0}^{C_{\lambda}^{\mathrm{ss}}} \cdot w_{\beta} \cdot \lambda_{\beta}\right\rangle=\left\langle\lambda_{\alpha}, w_{0} \cdot \lambda_{\beta}\right\rangle \quad$ for $1 \leq \alpha, \beta \leq N$ 
and

$$
\sum_{\alpha \neq \beta}\left\langle\eta_{\alpha}^{\perp},-w_{0}^{C_{\lambda}^{\mathrm{ss}}} \cdot \eta_{\beta}^{\perp}\right\rangle=\sum_{\alpha=1}^{N}\left|\eta_{\alpha}^{\perp}\right|^{2}
$$

If $\eta_{\alpha}^{\perp}=0$ for $1 \leq \alpha \leq N$ then $w_{0}^{C_{\lambda}^{\mathrm{ss}}} \eta_{\alpha}=\eta_{\alpha}$ for all $\alpha$, since $w_{0}^{C_{\lambda}^{\mathrm{ss}}} \lambda=\lambda$. This together with equality (3.6) imply that

$$
\lambda=\sum_{\alpha=1}^{N} \eta_{\alpha}^{\top}=\sum_{\alpha=1}^{N} \eta_{\alpha}=\sum_{\alpha=1}^{N} w_{\alpha} \cdot \lambda_{\alpha}
$$

and

$$
\left\langle\lambda_{\alpha}, w_{0} \cdot \lambda_{\beta}\right\rangle=\left\langle\eta_{\alpha}, w_{0}^{C_{\lambda}^{\mathrm{ss}}} \cdot \eta_{\beta}\right\rangle=\left\langle\eta_{\alpha}, \eta_{\beta}\right\rangle=\left\langle w_{\alpha} \cdot \lambda_{\alpha}, w_{\beta} \cdot \lambda_{\beta}\right\rangle \text { for } 1 \leq \alpha, \beta \leq N
$$

which is exactly what we need.

If $\eta_{N}^{\perp} \neq 0$ then Proposition 3.1 implies that

$$
-w_{0}^{C_{\lambda}^{\mathrm{ss}}} \eta_{N}^{\perp}=\sum_{\alpha=1}^{N-1} \eta_{\alpha}^{\perp}
$$

and

$$
\left\langle\eta_{\alpha}^{\perp}, \eta_{\beta}^{\perp}\right\rangle=\left\langle\eta_{\alpha}^{\perp}, w_{0}^{C_{\lambda}^{\mathrm{ss}}} \cdot \eta_{\beta}^{\perp}\right\rangle \text { for } 1 \leq \alpha, \beta \leq N-1
$$

from which we deduce

$$
\left\langle\eta_{\beta}^{\perp}, \eta_{N}^{\perp}\right\rangle=\left\langle\eta_{\beta}^{\perp},-w_{0}^{C_{\lambda}^{\mathrm{ss}}} \cdot \sum_{\alpha=1}^{N-1} \eta_{\alpha}^{\perp}\right\rangle=-\left\langle\eta_{\beta}^{\perp}, \sum_{\alpha=1}^{N-1} \eta_{\alpha}^{\perp}\right\rangle=\left\langle\eta_{\beta}^{\perp}, w_{0}^{C_{\lambda}^{\mathrm{ss}}} \eta_{N}^{\perp}\right\rangle .
$$

So for $1 \leq \alpha, \beta \leq N$, we have

$$
\left\langle\eta_{\alpha}, \eta_{\beta}\right\rangle=\left\langle\eta_{\alpha}, w_{0}^{C_{\lambda}^{\mathrm{ss}}} \cdot \eta_{\beta}\right\rangle
$$

and

$$
\left\langle\lambda_{\alpha}, w_{0} \cdot \lambda_{\beta}\right\rangle=\left\langle\eta_{\alpha}, w_{0}^{C_{\lambda}^{\mathrm{ss}}} \cdot \eta_{\beta}\right\rangle=\left\langle\eta_{\alpha}, \eta_{\beta}\right\rangle=\left\langle w_{\alpha} \cdot \lambda_{\alpha}, w_{\beta} \cdot \lambda_{\beta}\right\rangle
$$


On the other hand, $\eta_{N}^{\top} \in \mathfrak{z} \lambda$ implies $w_{0}^{C_{\lambda}^{\text {ss }}} \eta_{N}^{\top}=\eta_{N}^{\top}$, which means

$$
\begin{aligned}
\lambda & =\sum_{\alpha=1}^{N} \eta_{\alpha}^{\top}+0 \\
& =\sum_{\alpha=1}^{N-1} \eta_{\alpha}^{\top}+w_{0}^{C_{\lambda}^{\mathrm{ss}}} \eta_{N}^{\top}+\sum_{\alpha=1}^{N-1} \eta_{\alpha}^{\perp}+w_{0}^{C_{\lambda}^{\mathrm{ss}}} \eta_{N}^{\perp} \\
& =\sum_{\alpha=1}^{N-1} \eta_{\alpha}+w_{0}^{C_{\lambda}^{\mathrm{ss}}} \eta_{N} \\
& =\sum_{\alpha=1}^{N-1} w_{\alpha} \cdot \lambda_{\alpha}+w_{0}^{C_{\lambda}^{\mathrm{ss}}} w_{N} \cdot \lambda_{N}
\end{aligned}
$$

and

$$
\left\langle\lambda_{\alpha}, w_{0} \cdot \lambda_{N}\right\rangle=\left\langle\eta_{\alpha}, w_{0}^{C_{\lambda}^{\mathrm{ss}}} \cdot \eta_{N}\right\rangle=\left\langle w_{\alpha} \cdot \lambda_{\alpha}, w_{0}^{C_{\lambda}^{\mathrm{ss}}} \cdot w_{N} \cdot \lambda_{N}\right\rangle \cdot
$$

So the proof of Theorem 3.1 will be completed if we replace $w_{N}$ by $w_{0}^{C_{\lambda}^{\mathrm{ss}}} \cdot w_{N}$.

Corollary 3.1. Suppose

$$
\lambda \in \Delta_{N}
$$

then

$$
\left\langle\lambda, \sum_{\alpha=1}^{N} \lambda_{\alpha}\right\rangle \geq \sum_{\alpha=1}^{N}\left|\lambda_{\alpha}\right|^{2}+\sum_{\alpha \neq \beta}\left\langle\lambda_{\alpha}, w_{0} \cdot \lambda_{\beta}\right\rangle
$$

Moreover, there are two cases for the equality to hold:

1. Suppose $\lambda \neq 0$, then all but at most one of $\lambda_{\alpha}$ lie in $\mathfrak{z}$.

2. Suppose $\lambda=0$, then all but at most two of $\lambda_{\alpha}$ 's lie in $\mathfrak{z}$.

Proof. By adding up inequalities (2.1) and (1.2), we obtain

$$
\left\langle\lambda, \sum_{\alpha=1}^{N} \lambda_{\alpha}\right\rangle \geq \sum_{\alpha=1}^{N}\left|\lambda_{\alpha}\right|^{2}+\sum_{\alpha \neq \beta}\left\langle\lambda_{\alpha}, w_{0} \cdot \lambda_{\beta}\right\rangle .
$$

Now suppose the equality holds, there are two cases: 
If $\lambda \neq 0$, then the identities $\lambda=\sum \lambda_{\alpha}$ and $\left\langle\lambda_{\alpha}, w_{0} \cdot \lambda_{\beta}\right\rangle=\left\langle\lambda_{\alpha}, \lambda_{\beta}\right\rangle$ for all $\alpha, \beta$ implies that all but one of $\lambda_{\alpha}$ 's lie in $\mathfrak{z}$.

If $\lambda=0$, without loss of generality we may assume $\lambda_{N} \neq 0$, then the inequality

$$
0 \geq \sum_{\alpha=1}^{N}\left|\lambda_{\alpha}\right|^{2}+\sum_{\alpha \neq \beta}\left\langle\lambda_{\alpha}, w_{0} \cdot \lambda_{\beta}\right\rangle
$$

is equivalent to

$$
2\left\langle\lambda_{N}, \sum_{\alpha=1}^{N-1} \lambda_{\alpha}\right\rangle \geq\left|\lambda_{N}\right|^{2}+\sum_{\alpha=1}^{N-1}\left|\lambda_{\alpha}\right|^{2}+\sum_{\substack{\alpha \neq \beta \\ 1 \leq \alpha, \beta \leq N-1}}\left\langle\lambda_{\alpha}, w_{0} \cdot \lambda_{\beta}\right\rangle
$$

which can be obtained by adding up inequality (2.1) and (3.7) with $N$ replaced by $N-1$. Now the equality would imply both (2.1) and (3.7) become equality, the assumption $\lambda_{N} \neq 0$ would then reduces this case to the previous one.

In particular, if we let the group $G=U(r)$, hence $\mathfrak{W}=\mathfrak{S}_{r}$, the permutation group of $r$ letters and $w_{0}=\left(\begin{array}{l}12 \cdots(r-1) r \\ r(r-1) \cdots 21\end{array}\right) \in \mathfrak{S}_{r}$. Then Corollary 3.1 implies

Corollary 3.2. For any rank $r$ Hermitian matrix $A$, let $\lambda(A):=\left(\lambda_{1}\right.$ $\left.(A), \ldots, \lambda_{r}(A)\right) \in \mathbb{R}^{r}$ be the spectrum of $A$ with

$$
\lambda_{1}(A) \geq \lambda_{2}(A) \geq \cdots \geq \lambda_{r}(A) .
$$

Suppose $A_{1}, A_{2}, \ldots, A_{N}$ are Hermitian matrices satisfying

$$
\sum_{\alpha=1}^{N} A_{\alpha}=0
$$

then

$$
\sum_{\alpha=1}^{N} \lambda\left(A_{\alpha}\right)^{2} \leq \sum_{1 \leq \alpha \neq \beta \leq N} \lambda\left(A_{\alpha}\right) \lambda\left(-A_{\beta}\right) .
$$

Moreover, the equality holds if and only if all but possibly two of $A_{\alpha}$ 's are scalar matrices.

Finally, let us finish this section by an example that the equality holds. 
Example 3.1. Let

$$
A=\left[\begin{array}{ll}
a_{1} & \\
& a_{2}
\end{array}\right], B=\left[\begin{array}{ll}
b_{2} & \\
& b_{1}
\end{array}\right], C=\left[\begin{array}{ll}
c_{1} & \\
& c_{2}
\end{array}\right]
$$

such that $A+B+C=0$ and $a_{1} \geq a_{2}, b_{1} \geq b_{2}$ and $c_{1} \geq c_{2}$. Let $\lambda(A):=$ $\left(a_{1}, a_{2}\right), \lambda(B):=\left(b_{1}, b_{2}\right), \lambda(C):=\left(c_{1}, c_{2}\right)$ and

$$
\begin{aligned}
w_{0}: \mathbb{R}^{2} & \longrightarrow \mathbb{R}^{2} \\
(x, y) & \longmapsto(y, x)
\end{aligned}
$$

be the permutation of two elements then

$$
\begin{aligned}
|\lambda(A)|^{2}+|\lambda(B)|^{2}+|\lambda(C)|^{2}+2\left\langle\lambda(A), w_{0} \cdot \lambda(B)\right\rangle \\
\quad+2\left\langle\lambda(C), w_{0} \cdot \lambda(B)\right\rangle+2\left\langle\lambda(A), w_{0} \cdot \lambda(C)\right\rangle \\
=2\left(a_{1}-a_{2}\right)\left(c_{2}-c_{1}\right) \leq 0
\end{aligned}
$$

since $c_{1} \geq c_{2}$. Moreover the equality holds if and only if $a_{1}=a_{2}$ or $c_{1}=c_{2}$, without loss of generality, let us assume $a_{1}=a_{2}$. If we write $A+B=-C$ then $\lambda(-C)=\lambda(A)+\lambda(B)$ and $\left\langle\lambda(B), w_{0} \cdot \lambda(A)\right\rangle=\langle\lambda(B), \lambda(A)\rangle$, this will correspond to the first case in the Corollary 3.2. On the other hand if we write $A+B+C=0$, then we have $\lambda(A)+w_{0} \cdot \lambda(B)+\lambda(C)=0$ and

$$
\begin{aligned}
\left\langle\lambda(A), w_{0} \cdot \lambda(B)\right\rangle & =\left\langle\lambda(A), w_{0} \cdot \lambda(B)\right\rangle \\
\left\langle\lambda(A), w_{0} \cdot \lambda(C)\right\rangle & =\langle\lambda(A), \lambda(C)\rangle \\
\left\langle\lambda(C), w_{0} \cdot \lambda(B)\right\rangle & =\left\langle\lambda(C), w_{0} \cdot \lambda(B)\right\rangle .
\end{aligned}
$$

This will correspond to the second case in the Corollary 3.2.

\section{Acknowledgment}

Both authors are partially supported by RGC grants CUHK 403106 from Hong Kong government.

\section{References}

[1] M.F. Atiyah, Convexity and commuting Hamiltonians, Bull. London Math. Soc. 14(1) (1982), 1-15.

[2] A. Berenstein and R. Sjamaar, Coadjoint orbits, moment polytopes, and the Hilbert-Mumford criterion, J. Amer. Math. Soc. 13(2) (2000), $433-466$. 
[3] W. Fulton, Eigenvalues of sums of Hermitian matrices (after A. Klyachko), Seminaire Bourbaki, Vol. 1997/98. Astérisque No. 252 (1998), Exp. No. 845, 5, 255-269.

[4] V. Guillemin and S. Sternberg, Convexity properties of the moment mapping. Invent. Math. 67(3) (1982), 491-513.

[5] A.A. Klyachko, Equivariant bundles on toral varieties, Math. USSR Izvestiya, 35(2) (1990), 337-375.

[6] A.A. Klyachko, Stable bundles, representation theory and Hermitian operators, Selecta Math. 4 (1998), 419-445.

[7] N.C. Leung and X. Wang, A quadratic inequality of eigenvalues of Hermitian matrices satisfying $A+B=C$. Preprint.

Institute of Mathematical Sciences and Department of Mathematics The Chinese University of Hong Kong

HONG KONG

Department of Mathematics

The Chinese University of Hong Kong

HONG KONG

RECEIVED July 20, 2008 